\title{
Operation of TFTR Neutral Beams with Heavy Ions
}

J. H. Kamperschroer, T. N. Stevenson, K. E. Wright, L. E. Dudek, L. R, Grisham,

R. A. Newman, T. E. O'Connor, M. E. Oldaker, A. von Halle, and M. D. Williams

Princeton Plasma Physics Laboratory

Princeton University, Princeton, $\mathrm{NJ} 08543$

\begin{abstract}
High $\mathrm{Z}$ neutral atoms have been injected into TFTR plasmas in an attempt to enhance plasma confinement through modification of the edge eiectric field. TFTR ion sources have extracted $9 \mathrm{~A}$ of $62 \mathrm{keV} \mathrm{Ne}$ for up to $0.2 \mathrm{~s}$ during injection into deuterium plasinas, and for $0.5 \mathrm{~s}$ during conditioning pulses. Approximately $400 \mathrm{~kW}$ of $\mathrm{Ne}^{0}$ have been injected from each of two ion sources. Operation was at full bending magnet current, with the $\mathrm{Ne}^{+}$barely contained on the ion dump. Beamline design modifications to permit operation up to $120 \mathrm{keV}$ with krypton or xenon are described. Such ions are too massive to be deflected up to the ion dump. The plan, therefore, is to armor those components receiving these ions. Even with this armor, modest increases in the bending magnet current capability are necessary to safely reach $120 \mathrm{kV}$ with $\mathrm{Kr}$ or Xe. Information relevant to heavy ion operation was also acquired when several ion sources were inadvertently operated with water contamination. Spectroscopic analysis of certain pathological pulses indicate that up to $6 \%$ of the extracted ions were water. After dissociation in the neutralizer, water yields oxygen ions which, as with $\mathrm{Ne}, \mathrm{Kr}$, and $\mathrm{Xe}$, are underdeflected by the magniet. Damage to a calorimeter scraper, due to the focal properties of the-magnet, has resulted. A magnified power density of $6 \mathrm{~kW} / \mathrm{cm}^{2}$ for $2 \mathrm{~s}$, from $-90 \mathrm{~kW}$ of $\mathrm{O}^{+}$, is the suspected cause.
\end{abstract}




\section{Introducion}

Preliminary experiments have been carried out on both TFTR and DIII- $\mathrm{D}^{1}$ using beams of heavy atoms to generate a radial electric field at the edge of the plasma. Such fields, created by a biased probe, have been shown to reduce plasma transport. ${ }^{2}$ Techniques have been proposed by Ohkawa $^{3}$ and Zarnstorff ${ }^{4}$ to create similar fields through the injection of heavy atoms. If a heavy atom is multiply ionized by the plasma, and the resulting ion exits the plasma, a net charge and, hence, an electric field can be induced over the distance the particles penetrate the plasma. To test this hypothesis, argon beams have been injected into DIII-D and neon beams into TFTR.

One of the difficulties associated with using heating neutral beam systems for this task is that they were designed for hydrogenic gases. As a result, the magnets cannot bend heavy ions to the ion dumps. The IFTR magnet was designed to handle ions no stiffer than $120 \mathrm{keV} \mathrm{T}{ }_{3}{ }^{+}$, or $120 \mathrm{kG}-\mathrm{cm}$. A goal to achieve $120 \mathrm{keV} \mathrm{Xe}$, or $570 \mathrm{kG}-\mathrm{cm}$, has, however, been set. An additional problem is that the TFTR magnet acts as a focussing lens. Even the modest power levels, of order one hundred kilowatts, associated with the heavy ions can cause thermal problems due to enhanced power densities. This was demonstrated when several TFTR ion sources operated for a short period of time with small water leaks.

TFTR neutral beams have injected $60 \mathrm{keV}$ neon atoms for short pulses with no deleterious effect on the beamline. Information regarding operation of heavy ions has been obtained from the analysis of the cperation of water contaminated sources. Acceleration of water ions, and their subsequent dissociation in the neutralizer, results in the deflection of $\mathrm{O}^{+}$with an eneigy of $\sim 90 \%$ of the acceleration poteritial. 
Operation with 80 to $120 \mathrm{kV}$ krypton or xenon beams will necessitate placing armor in those locations where ion power will be deposited. This is necessary since the repository for this power is not the ion dump, but rather components not designed to handle direct beam. Increasing the field of the magnet is also being examined in a attempt to increase beam energy for which such operation is feasible.

\section{2) Neon Beam Operation}

Neon beams were run at $60 \mathrm{kV}, 8$ to $10 \mathrm{~A}$, for puise lengths up to $500 \mathrm{~ms}$ during conditioning and $200 \mathrm{~ms}$ during injection. The six ion sources on the two counter-injection beamlines all ran with neon. One accelerator failed during $\mathrm{Ne}$ conditioning due to a ruptured gradient grid rail. Speculation is that this was caused by too much gas or a pre-existing defect in the accelerator structure. Subsequent operation of the other five sources was flawless. Two sources, the one with the largest tangency radius on each counter-injecting beardine, injected 60 $\mathrm{keV}$ neon atoms into TFTR. Neon gas was injected into both the ion source and the neutralizer. $20 \%$ of the throughput was to the source, the remainder went into the neutralizer to ensure sufficient line density for neutralization.

$60 \mathrm{keV} \mathrm{Ne}{ }^{+}$is the limit of stiffness $(160 \mathrm{kG}-\mathrm{cm}$ ) which can be accommodated on the full-energy ion dump. The magnet power supply was operated at full current, $1000 \mathrm{~A}$, with Ne ions barely deflected to the bottom of the ion dump. The deflection angle was $40^{\circ}$, as compared to the nominal $60^{\circ}$ for full-energy deuterium ions. Normally the focal point of the magnet, for $60^{\circ}$ deflection, is at the exit of the magnet, providing the ion beam time to spread out before reaching the dump. At $40^{\circ}$ the focal length is longer; the focal point falling closer to the ion dump. 
Figure 1 shows the trajectories of deuterium ions and $\mathrm{O}^{+}$for normal operation. For each trajectory, the focal point of the magnet is indicated. Due to the curvature of the fringing field at the entrance to the magnet, and the $45^{\circ}$ inclination of the magnet, positive ions experience a force toward the beam axis as they enter the magnet, and, hence, are focussed. Ions stiffer than half-energy $\mathrm{D}^{+}$ are defocussed upon exiting the magnet. The stiffer the ion the greater the defocussing, with the net effect that the focal point recedes from the magnet as stiffness increases. $\mathrm{Ne}^{+}$trajectories are similar to those of $\mathrm{D}_{3}{ }^{+}$; both having a magnification of eight at the ion dump. Fortunately, the neon ion power is reduced due to the lower operating voltage, the reduced perveance of the higher mass ion, and the higher neutralization efficiency. 4 Approximately $400 \mathrm{~kW}$ of 60 $\mathrm{keV} \mathrm{Ne}{ }^{0}$ was injected into the torus per ion source and about $80 \mathrm{~kW}$ of $\mathrm{Ne}^{+}$was dissipated on the ion dump. An ion dump power density of $\sim 5 \%$ of that for high power deuterium operation resulted. Temperature rises on the ion dump during neon operation were less than $-5^{\circ} \mathrm{C}$.

Nominal divergences perpendicular to the rails for neon beams were $1.2^{\circ}$ to $1.4^{\circ}$, equivalent to deuterium. Neon beams were observed spectroscopically, ${ }^{5}$ to search for the extraction of Aeuterium and $\mathrm{Ne}^{++}$. Neither was found to within the measurement accuracy of the instrument. The inferred $\mathrm{Ne}^{++}$fraction of the beam was less than $2 \%$, as expected. ${ }^{6}$

\section{3) $\mathrm{H}_{2} \mathrm{O}$ Contamination}

The initial symptom of water contamination of the ion sources was a ramping of the arc voltage as a function of time, especially late in the pulse. This effect was later correlated with Doppler-shifted $\mathrm{H}_{\alpha}$ emission from particles with an energy 
approximately $1 / 18$ that of the full acceleration potential; corresponding to the energy of a hydrogen atom created from the dissociation of water. As pulse length increased, it was noticed that the relative intensity of the water-related signal increased, implying a time varying source of water. Four ion sources had measurable quantities of water in the beam. After removal and inspection of these sources, it was found that inadequately cooled Langmuir probes in the arc chamber were the culprit. As the probes got hot during the discharge, a crack in the metal to ceramic seal opened and released water. The size of the crack was a function of temperature, and, hence, time. Figure 2 shows the increase in extracted water fraction, for the worst ion source of record, as a function of pulse length.

Figure 3 is the worst case contaminated $D_{\alpha}$ spectra for which spectroscopic data was collected. Both the data points and a least squares fit to the data are indicated. ${ }^{7}$ Left to right, the peaks are: full-energy $D^{\circ}$, half-energy $D^{\circ}$, thirdenergy $\mathrm{D}^{0}$, and then water. Half- and third-energy $\mathrm{D}^{0}$ are products of the dissociation and reutralization of $\mathrm{D}_{2}{ }^{+}$and $\mathrm{D}_{3}{ }^{+}$, respectively. Not shown is the unshifted $D_{\alpha}$ line, which lies to the right of the water signal. The accelerating potential was $95 \mathrm{kV}$, the beam current $40 \mathrm{~A}$, the pulse length $1.4 \mathrm{~s}$, the calculated $\mathrm{D}^{+}, \mathrm{D}_{2}{ }^{+}, \mathrm{D}_{3}{ }^{+}$fractions of the extracted beam were $71 \%, 20 \%, 3 \%$. The fraction of the extracted current attributable to water was $6 \%$. Since the measurement is integrated over the beam pulse, and the water level was found to be increasing, approximately linearly, with time, the extracted water content at the end of the pulse is $-12 \%$. Pulse lengths up to $2 \mathrm{~s}$ were run under these contaminated conditions, implying that even greater water concentrations were most likely created, but not observed. Spectroscopic data was acquired for only a few of the dozens of long pulse shots. 
Accelerated water ions dissociate in the neutralizer, producing hydrogen and oxygen ions and atoms. Dissociation is assumed to be nearly $100 \%$ complete at the line densities of the TFTR neutralizer. Oxygen dissociation fragments carry away $89 \%$ of the energy of the water and each hydrogenic fragment receives $5.5 \%$. Oxygen is neutralized in the deuterium gas cell with an equilibrium neutral fraction of nearly $80 \%{ }^{6,8}$ For the case of figure 3 , the time averaged oxygen ion power is $40 \mathrm{~kW}$, a power level generally of no concern.

The problem is that oxygen ions are considerably stiffer than deuterium ions of comparable energy, and hence are under-deflected. Figure 1 shows the locations of the focal points of the magnet for ions of different relative stiffness. Trajectories for $D^{+}(E / 3), D^{+}(E / 2), D^{+}(E), D_{2}{ }^{+}(2 E / 3), D_{2}{ }^{+}(E)$, and $D_{3}{ }^{+}(E)$ were computed with BEAM. ${ }^{9}$ From these focal lengths, that for oxygen was extrapolated. Due to the proximity of the focal point to the scraper, the magnification of the $\mathrm{O}^{+}$at the calorimeter scraper is 25 .

Upon completion of the operating period during which water contaminated operation took place, it was noticed that the calorimeter scraper (curved scraper just before the calorimeter) was melted. A melt spot ran from the top of the scraper, where it was deepest, to about $2 / 3$ of the way to the bottom, where it disappeared. From the tens of centimeter long, $\sim 2 \mathrm{~cm}$ wide shape of the melt spot, the focal properties of the magnet were suspected as the cause of the problem. Based upon the pattern of shadowed components behind the scraper, it was estimated that the angle of the under-deflected particles was $-17^{\circ}$. Given that the bottom of the melt spot just reached the melting temperature of copper, that the angle of incidence at this point was $35^{\circ}$, and that the angle of incidence at the top of the scraper was $59^{\circ}$, we deduce a surface temperature (scraper is $3 / 4 "$ thick) at the top of the scraper of $1600^{\circ} \mathrm{C}$. 
Carslaw and Jaeger ${ }^{10}$ have solved the one dimensional hest transfer problem for a plate of finite thickness, with no heat removal out the back, and with a linearly increasing heat flux. For the temperature as a function of depth, z, through the plate of thickness $d$ they give

$$
T(z)=\frac{8 F_{0} \sqrt{\gamma} t^{1.5}}{k} \sum_{n=0}^{\infty}\left\{i^{3} \operatorname{erfd}\left[\frac{(2 n+1) d-z}{2 \sqrt{\gamma}}\right]+i^{3} \operatorname{erfd}\left[\frac{(2 n+1) d+z}{2 \sqrt{\gamma}}\right]\right\}
$$

$F_{0}$ is the heat flux at one second, $k$ the thermal conductivity, $\gamma$ the thermal diffusivity, and the time. $i^{3}$ ersc is the a repeated integral of the error function. ${ }^{11}$

For the case of figure $3, F_{0}$ is $3 \mathrm{~kW}^{\prime} \mathrm{cm}^{2}, F_{0}$ is calculated from the extracted ion power, the water fraction, and the equilibrium $\mathrm{O}^{+}$fraction of the neutralizer. Figure 4 shows the computed surface temperature versus time. The temperature rises to a value slightly in excess of $1600^{\circ} \mathrm{C}$. Through the focussing action of the magnet, a. modest power density of $180 \mathrm{~W} / \mathrm{cm}^{2}$ (at $1.4 \mathrm{~s}$ ), has been concentrated to $\sim 6 \mathrm{~kW} / \mathrm{cm}^{2}$ (at the end of the pulse). Focussing, together with pulse lengths of $\sim 2$ $s$, cause temperatures in excess of the melting temperature of copper. Intentional operation with heavy ions must take this effect into account.

Further evidence of the focussed $\mathrm{O}^{+}$power was obtained from thermocouples on the full-energy ion dump. A portion of the oxygen ions strike the bottom of the ion dump. These are also focussed, but the magnification is reduced since the point of impact is farther from the focal point. The profile obtained from the vertical column of 12 thermocouples on the durnp changed as a function of time. As the pulse length was increased, the bottom of the dump increased in temperature faster than the rest of the dump, implying that the bottum portion of the beam was increasing with time. 


\section{4) Krotor and Xenon}

Greate- radial electric fields can be created by heavier ions due to their higher atomic number. For this reason, operation with krypton and xenon are of interest. At $120 \mathrm{keV}$ such ions are stiffer (by 3 to 3.5 times) than $60 \mathrm{keV} \mathrm{Ne}^{+}$. As with oxygen, these beam are expected to be well neutralized. Also, due to the large masses involved, the extracted currents will be no more than $10 \mathrm{~A}$ at $120 \mathrm{kV}$.

Two approaches are being pursued to reach the goal of $120 \mathrm{keV}$ Xe. The first is to armor those components in the beamline exposed to the ions; the second is to increase the magnetic field. Given the $1000 \mathrm{~A}$ limit of the magnet power supply and the requirement that heavy ions be kept out of the drift duct, $80 \mathrm{keV} \mathrm{Kr}$ operation is possible. The resultant deflection angle will be $13^{\circ}$, slightly less than the $17^{\circ}$ of $\mathrm{O}^{+}$. As with $\mathrm{O}^{+}$, the central ray of the beam is incident upon the calorimeter scraper, but the focal point has moved $-30 \mathrm{~cm}$ behind the scraper, yielding a magnification of 5 . Armoring is required beneath the calorimeter, when it is raised for injection, and above the calorimeter exit scraper.

Pulsed operation of the magnet and paralleling power supplies are being considered as means to increase the operating magnetic field. An effort is under way to examine these possibilities and quantify difficulties with regard to magnet saturation and heating. If the current can be increased to $1200 \mathrm{~A}, 120 \mathrm{keV} \mathrm{Kr}{ }^{+}$ can be deflected by $13^{\circ}$, and hence to the armor described above. At $1500 \mathrm{~A}, 120$ $\mathrm{keV}, \mathrm{Xe}^{+}$becomes a possibility.

Approximately $200 \mathrm{~kW}$ of ion power must be dissipated for $120 \mathrm{keV} \mathrm{Kr}$. The highest power density will be at the calorimeter scraper and will be $-1.6 \mathrm{~kW} / \mathrm{cm}^{2}$. Surface temperature rises of $400^{\circ} \mathrm{C}$ are predicted for $0.5 \mathrm{~s}$ pulses. Reducing the angle of incidence on this scraper for added safety margin is being considered. 
9

An additional problem associated with krypton and xenon is their effect on the cryogenic pumping system. Krypton freezes at $100 \mathrm{~K}$, xenon at $140 \mathrm{~K}$. Regeneration of xenon will most likely require the liquid helium panel temperature be elevared to liquid nitrogen temperature for many hours to remove the xenon. Experiments are planned to quantify the extent of this difficulty.

\section{Acknowledonnents}

We would like to thank all members of the TFTR neutral beam operations team for their continued superior performance. This work was supported by the U. S. Department of Energy under contract DE-AC02-76-CHO-3073. 


\section{References}

${ }^{1}$ R-M. Hong, J. Kim, private communication.

${ }^{2}$ R. J. Taylor, M. L. Brown, B. D. Fried, H. Grote, J. R. Liberati, G. J. Morales, P. Pribyl, D. Darrow, and M. Ono, Phys. Rev. Lett. 63. 2365 (1989).

${ }^{3}$ T. Ohkawa and R. L. Miller, Nucl. Fusion 30, 1616 (1990).

${ }^{4}$ M. C. Zamstorff, private communication.

5. H. Kamperschroer, H. W. Kugel, M. A. Reale, S. L. Hayes, G. A. Johnson, J.

L. Lowranze, P. A. Shah, P. Sichta, B. W. Sleaford, M. D. Williams, and P. M. Zucchino, Rev. Sci. Instrum. 58, 1362 (1987).

${ }^{6}$ C. F. Barnett, J. A. Ray, E. Ricci, M. I. Wilker, E. W. McDaniel, E. W. Thomas, and H. B. Gilbody, Oak Ridge National Laboratory Report ORNL-5206 (Feb. 1977). ${ }^{7}$ C. F. Burrell, W. S. Cooper, W. F. Steele, and R. R. Smith, in Proceedings of the Seventh Symposium on Engineering Problems of Fusion Research, Knoxville, 1977 (IEEE, New York, 1977), Vol. 1, p. 374.

${ }^{8}$ C. F. Barnett, J. A. Ray, and J. C. Thomoson, Oak Ridge National Laboratory Report ORNL-3113 (Aug. 1964).

${ }^{9} \mathrm{~K}$. Halbach, computer code BEAM (Lawrence Berkeley Laboratory, Berkeley, CA).

${ }^{10} \mathrm{H}$. S. Carslaw and J. C. Jaeger, Conduction of Heat in Solids, 2nd ed. (Oxford University Press, London, 1959), p. 113.

${ }^{11 S e e, ~ f o r ~ e x a m p l e, ~ W . ~ G a u t s c h i, ~ " E r r o r ~ F u n c t i o n s ~ a n d ~ F r e s n e l ~ I n t e g r a l s ", ~ i n ~}$ Handbook of Mathemazical Functions with Formulas, Graphs, and Mathematical Tables. edited by M. Abramowitz and I. A. Stegun (U. S. Government Printing Office, Washingtor, D. C., 1972). 
Figure Captions

Figure 1. Elevation schematic of a TFTR beamline showing trajectories of various ions. The dots on the trajectory indicate the location of the focal point for that trajectory.

Figure 2. The calculated water content of the most contaminated ion source as a function of pulse length.

Figure 3. OMA spectrum of a $1.4 \mathrm{~s}$ beam extraction from the most contaminated in source. The unshifted $D_{\alpha}$ line is off the right side of the figure.

Figure 4. Calculated surface temperature versus time for the case of figure 3 extrapolated to a two second pulse length. 


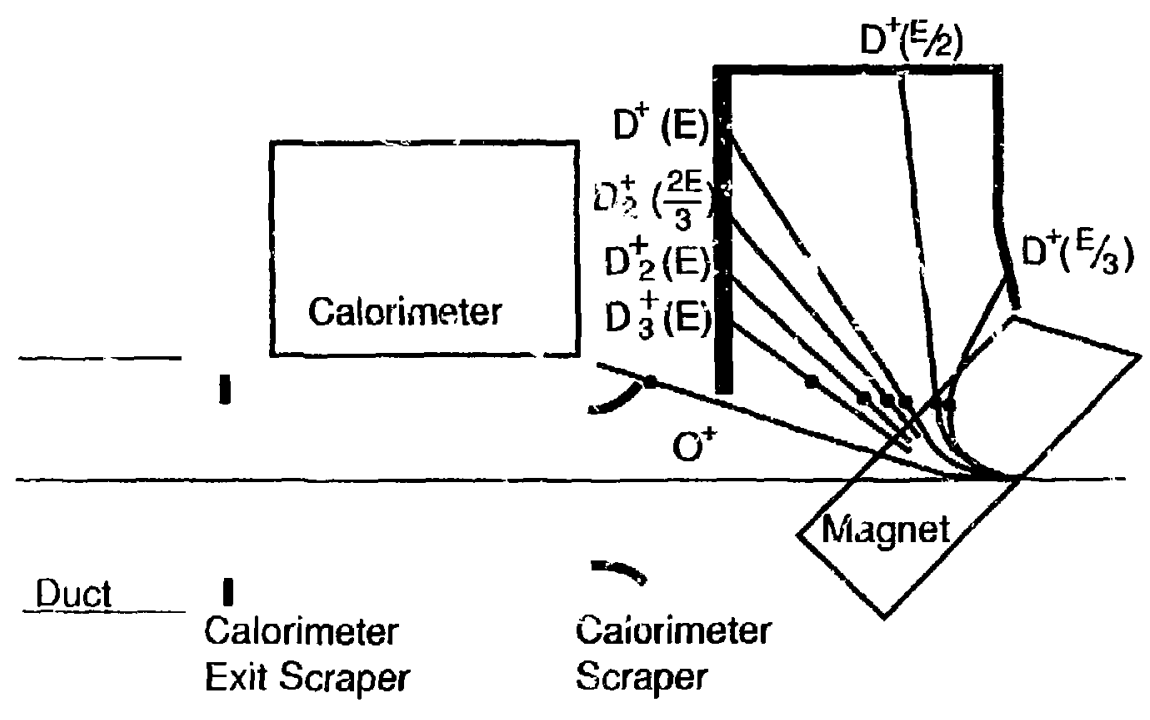




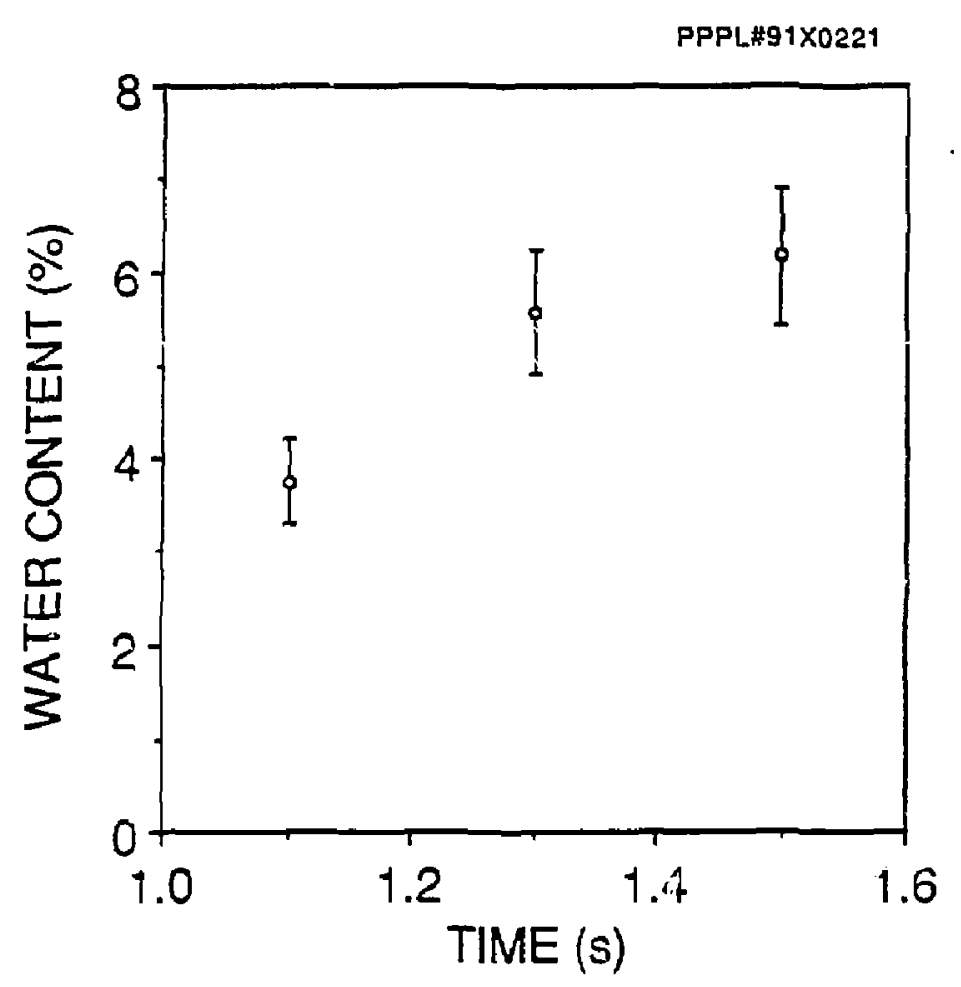

事 


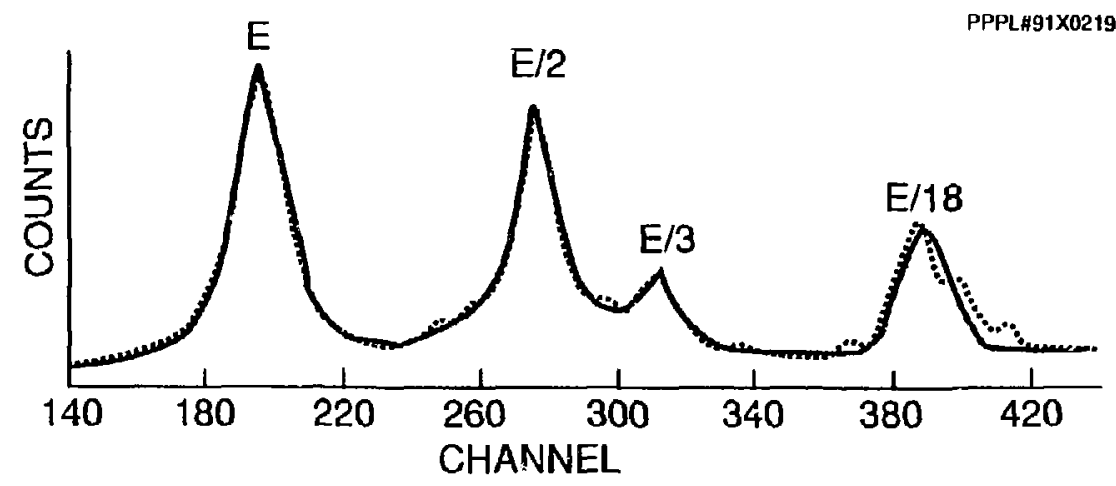




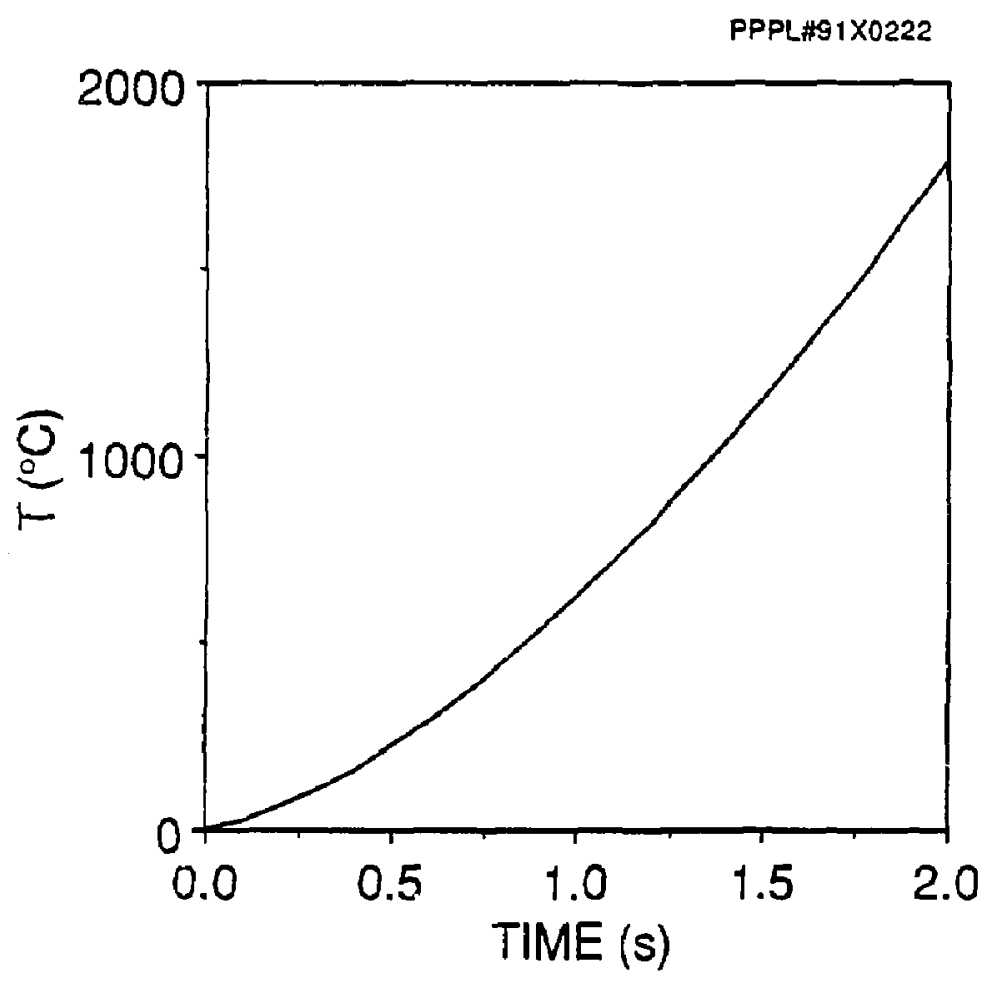

$\square$
$\vdots$
3
3
0 